\title{
Cambios estacionales en los ovarios de peces siluriformes: comparación de tres especies en un ambiente subtropical de Argentina
}

\author{
Nélida Marcela Romero ${ }^{1}$ y Rosa Vera-Mesones ${ }^{2}$ \\ ${ }^{1,2}$ Consejo de Investigación de la Universidad Nacional de Salta. Avda. Bolivia 5150 - 4400 - Salta, Argentina; \\ 'nmarceromero@hotmail.com; ${ }^{2}$ rosa_veramesones@yahoo.com.ar
}

Recibido 26-II-2010 Corregido 4-VI-2010 Aceptado 21-VI-2010

\begin{abstract}
Seasonal changes in the ovaries of three species of Siluriform fish: comparison of three species in a subtropical Argentinean environment. The Siluriforms are a diverse, widely distributed group of Ostariophysi fish, with their greatest diversity in the tropical and subtropical regions. The order includes an important group of species of small size, relevant in the trophic nets of rivers and streams of different magnitudes. In the present work, we analyzed the seasonal changes in the ovaries of smallsized Siluriforms from rivers and streams in Valle de Lerma, Salta Province, Argentina. Seasonal samplings were carried out from 1995 to 2004 in four different environments. The specimens collected were fixed and measured, and the gonads were removed for histological processing. The species selected were Corydoras paleatus, Trichomycterus spegazzinii and Heptapterus mustelinus. The parameters analyzed were the condition factor (K), the gonadosomatic index (GSI), ovocitary frequency, fecundity, minimum size at sexual maturity, and macro and microscopic characteristics of the gonads throughout the seasons. The spawning season was determined for each species. The histological changes in the different stages of maturity of the gonads are similar among these species. They are partial spawners; the spawning season extends from early spring to mid summer for T. spegazzinii and H. mustelinus, whereas C. paleatus spawn throughout the year, with peaks in summers.
\end{abstract}

\section{KEY WORDS}

Siluriforms, Corydoras paleatus, Trichomycterus spegazzinii, Heptapterus mustelinus, reproduction.

\section{RESUMEN}

El Orden Siluriformes constituye un grupo diverso y ampliamente distribuido de peces Ostariofísidos, cuya mayor diversidad está en la región tropical y subtropical. Incluye un importante grupo de especies de bajo porte cuya relevancia se relaciona con las redes tróficas de ríos y arroyos de diferentes magnitudes. En este trabajo se analizan los cambios estacionales de ovarios de peces Siluriformes de bajo porte frecuentes en ríos y arroyos del Valle de Lerma, Salta, Argentina. Se realizaron muestreos estacionales desde 1995 hasta 2004, en cuatro ambientes diferentes. Los ejemplares colectados fueron fijados y medidos, las gónadas se extirparon para su procesamiento histológico. Las especies seleccionadas fueron: Corydoras paleatus, Trichomycterus spegazzinii y Heptapterus mustelinus. Se analizaron: valor de condición K, índice gónado-somático, frecuencia ovocitaria, fecundidad, talla mínima de madurez sexual, características macroscópicas y microscópicas de las gónadas en las diferentes estaciones del año, determinándose en cada especie el período de desove. Los cambios histológicos en las diferentes etapas de madurez de las gónadas de estas especies son similares, son desovadoras parciales, la época de desove de T. spegazzinii y H. mustelinus se extendería desde la primavera temprana hasta mediados de verano, C. paleatus desovaría durante todo el año, con picos en verano.

PALABRAS CLAVE

Siluriformes, Corydoras paleatus, Trichomycterus spegazzinii, Heptapterus mustelinus, reproducción.
Es de amplio conocimiento que los primeros estadios de desarrollo son críticos para las fluctuaciones en la abundancia de los peces (Saborido 2004). Para maximizar el éxito reproductivo, un individuo debe repartir sus recursos energéticos adecuadamente y procurar que su descendencia eclosione en las condiciones ambientales favorables. Por lo tanto, la estacionalidad con que se produce el desove es de vital importancia para el futuro desarrollo y supervivencia de la descendencia (Saborido 2004).

Dentro de la fauna íctica se pueden reconocer una gran diversidad de estrategias reproductivas. Por lo tanto, el estudio de los cambios que ocurren en las gónadas de los 
peces puede aportar importantes conocimientos para determinar cuál es la estrategia reproductiva empleada.

Agostino et al. (1995) verificaron que algunas de las especies de peces de pequeño a mediano porte que habitan ríos y arroyos, desarrollan estrategias reproductivas que se caracterizan por presentar fecundación externa, desove múltiple, períodos reproductivos cortos y marcada estacionalidad reproductiva.

El Orden Siluriformes constituye el grupo más diverso y ampliamente distribuido de peces Ostariofisidos. La diversidad más alta se encuentra en la región tropical y subtropical, especialmente de Suramérica, África y Sudeste asiático (Monasterio de Gonzo 2003). Comprenden 36 familias, 412 géneros y más de 2800 especies (Montero \& Autino 2009), entre ellas 1440 han sido documentadas en América, la mayoría en aguas continentales. El 37,5\% de las especies correspondientes a la ictiofauna continental de Argentina son Siluriformes (Braz de Araujo \& Garutti 2002). En Argentina se registran 12 familias, 85 géneros y 193 especies (Monasterio de Gonzo et al. 2008)

Este Orden incluye especies de interés deportivo y económico, así como especies de bajo porte (menores de $12 \mathrm{~cm}$ de longitud estándar), cuya relevancia se relaciona fundamentalmente con las redes tróficas de ríos y arroyos de diferentes magnitudes (Braz de Araujo \& Garutti 2002).

Si bien el estudio de las características reproductivas es de vital importancia para entender la dinámica de las poblaciones de peces de relevancia económica, también se deben realizar este tipo de análisis en especies de bajo porte, ya que constituyen los primeros eslabones en las redes tróficas de estos ambientes. El objetivo de este trabajo fue analizar los cambios estacionales de las gónadas de hembras de Corydoras paleatus, Trichomycterus spegazzinii y Heptapterus mustelinus, tres especies de Siluriformes frecuentes en los ríos y arroyos del Valle de Lerma, Salta, Argentina.

\section{METODOLOGÍA}

\section{Área de estudio}

La provincia de Salta (noroeste de Argentina), se encuentra dentro de un área montañosa con clima subtropical con estación seca (invierno), debido a esto los sistemas fluviales muestran una fuerte fluctuación en los caudales de agua. Este estudio se llevó a cabo en ambientes acuáticos del Valle de Lerma de la Provincia de Salta.

\section{Ambientes estudiados}

Se realizaron muestreos estacionales en los siguientes ambientes lóticos del Valle de Lerma: paraje La Ciénega y arroyo Isasmendi del Río Arias (24 48' 92" S, 65 27,02'37" O, altitud 1198m.s.n.m.) departamento Capital, situados al oeste de la ciudad de Salta, de 1995 a 2001. Arroyo El Gallinato $\left(24^{\circ} 41^{\prime} 10,6^{\prime \prime} \mathrm{S}, 65^{\circ} 18^{\prime} 32,7^{\prime \prime} \mathrm{O}\right.$, altitud $1178 \mathrm{~m}$. s. n. m.) y río Mojotoro ( $24^{\circ} 42^{\prime} 54,8^{\prime \prime} S, 65^{\circ} 23^{\prime} 15,5^{\prime \prime} \mathrm{O}$, altitud 1210 m. s. n. m.) departamento La Caldera, en dirección NE de la ciudad de Salta, en el período 2002 a 2004 (Fig. 1).

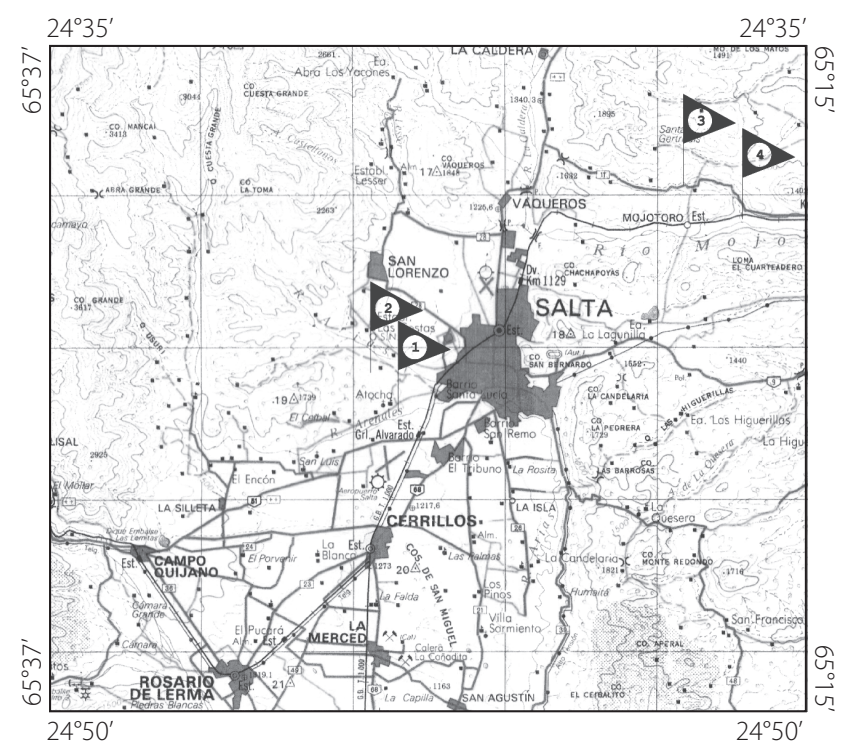

FIG. 1. Mapa de sitios de muestreo realizados en el Valle de Lerma, Salta, Argentina; (1) La Ciénega; (2) Arroyo Isasmendi; (3) Arroyo el Gallinato; (4) Río Mojotoro.

Fuente: Carta topográfica - Hoja 2566-II "Salta" Escala 1:250.000 - Año 1986

\section{Metodología de muestreo}

Se utilizaron como artes de pesca redes de copo con $5 \mathrm{~mm}$ de luz de malla, trampas artesanales y cañas con anzuelo. Los muestreos con redes y trampas se realizaron fundamentalmente en los brazos secundarios, preferentemente en las riberas vegetadas, donde la velocidad de la corriente es menor; los anzuelos se utilizaron en sitios de mayor corriente y profundidad. Todas las colectas se hicieron durante las primeras horas de la tarde, con un esfuerzo de muestreo de 30 minutos por sitio, realizado por tres personas. 


\section{Registro de datos}

Los peces recolectados en cada uno de los ambientes se fijaron in situ en formol al 10\%. En el laboratorio, los ejemplares se separaron por sitio y los individuos por especies fueron contados, además, se registró su peso total con las vísceras y su longitud estándar.

Para el procesamiento histológico se seleccionaron solamente los individuos con longitud estándar igual o mayor a $10 \mathrm{~mm}$, en los que se realizó la autopsia extirpando las gónadas, registrándose el peso y longitud de las mismas. Se utilizó calibre digital y balanza Sartorius con precisión de $0,1 \mathrm{~g}$.

Se calculó el factor de condición o bienestar (K) aplicando la siguiente fórmula (Nikolsky 1963):

$$
K=\frac{P t}{(L s t)^{3}} \times 100
$$

Siendo Pt el peso total y Lst la longitud estándar.

Para determinar el efecto de la madurez sexual sobre este factor se realizaron análisis de la varianza de dos vías (ANOVA) con $a=0,05$ y la prueba a posteriori de Tukey para las comparaciones múltiples de los promedios, usando el programa Info-Stat.

El índice gonadosomático (IGS) se obtuvo a partir de la siguiente fórmula (Calvo \& Dadone 1972):

$$
\mathrm{IGS}=\frac{\mathrm{Pg}}{\mathrm{Pt}} \times 100
$$

Siendo $\mathrm{Pg}$ el peso de la gónada.

Los ovarios extirpados se procesaron según técnicas histológicas de rutina, incluyéndose, en la mayoría de los casos, un ovario completo y reservándose el otro en formol. Las muestras se deshidrataron con una serie creciente de alcoholes y fueron incluidas en parafina. Se realizaron cortes longitudinales de la gónada, con espesor de 3 a $5 \mu \mathrm{m}$ en micrótomo de deslizamiento, los cuales fueron coloreados con tinciones de H\&E, PAS y tricrómico de Masson (Mc Quinn \& Landry 1988).

La frecuencia ovocitaria se determinó mediante la confección y análisis de histogramas, considerando los tamaños de los ovocitos, siguiendo el criterio de Saborido (2004). Estas medidas fueron tomadas de los cortes histológicos en los diferentes estadios de madurez, utilizando un microscopio Zeiss con ocular graduado en micras.

El cálculo de la fecundidad se realizó siguiendo el método gravimétrico de Bagenal (1978), tomando submuestras de $10 \mathrm{mg}$ del centro de ovarios maduros. Para establecer la fecundidad en cada especie se contaron los ovocitos maduros comprendidos en el rango de 1000 a $1500 \mu \mathrm{m}$ ( $C$. paleatus), 1000 a $1100 \mu \mathrm{m}$ (T. spegazzinii) y 1200 a $1900 \mu \mathrm{m}$ (H. mustelinus). Se aplicó la siguiente fórmula:

$$
\mathrm{F}=\mathrm{N} \times \frac{\mathrm{Pg}}{\mathrm{Ps}}
$$

Donde $N$ es el número total de ovocitos maduros del recuento; $P g$ el peso de la gónada en $m g$ y $P s$ el peso de la submuestras en $\mathrm{mg}$.

Se determinó la talla mínima de madurez sexual, considerando para ello la longitud estándar de la hembra de menor talla con ovario maduro.

Se analizaron las características macroscópicas y cambios histológicos de los ovarios en las diferentes estaciones del año. Según las características histológicas se establecieron las siguientes etapas de madurez gonadal: inmaduras, en maduración, maduras y desovadas; considerando el criterio y terminología de Yamamoto (1956), Vazzoler (1996) y Saborido (2004).

Se realizó la documentación fotográfica utilizando una cámara digital Sony de 7,2 mega pixeles.

\section{Aspectos generales de las especies}

Corydoras paleatus (Jenyns 1842). Conocida como"tachuela" o "limpia fondo" se encuentra habitualmente sobre el lecho, bajo las piedras u oculta entre la vegetación ribereña, es detritívora y considerada como biotransformadora (Ringuelet et al. 1967), característica que la han llevado a ubicarla como una especie muy apreciada para los acuarios y peceras (Werner 1993). El cuerpo presenta la parte ventral aplastada y parte dorsal bombeada; la aleta adiposa, se encuentra detrás de la aleta dorsal, el primer radio de la aleta pectoral está transformado en una gran espina que desempeña un papel importante en la reproducción. En lugar de escamas cuentan con placas óseas que se solapan como si fueran tejas que les cubren los flancos, la cabeza y la espalda; presenta un par de barbas en la mandíbula inferior, los ojos son grandes y móviles. Los machos alcanzan una longitud de hasta $50 \mathrm{~mm}$ y las hembras hasta $70 \mathrm{~mm}$ (Las Heras 2010). Son de comportamiento gregario. En Suramérica se encuentran distribuidos en la cuenca del río Paraná, en ríos de Uruguay y Brasil (Montero \& Autino 2009).

Trichomycterus spegazzinii (Berg 1897). Se caracteriza por estar presente en aguas corrientes, limpias y frías con lecho arenoso y pedregoso. En la región son conocidos como "bagres del torrente", "bagres anguilas" o "bagres serranos" (Ringuelet 1975). El cuerpo presenta papilas terminadas en punta y muy próximas entre ellas que le dan 
aspecto verrugoso, poseen manchas oscuras excepto en la zona ventral que es blanquecina. Alcanzan una longitud de hasta 130 mm (Mirande \& Aguilera 2009). Distribución en la provincia de Salta en cuencas de río Bermejo y Juramento (Monasterio de Gonzo 2003).

Heptapterus mustelinus (Valenciennes 1835). Se lo conoce vulgarmente como "yusca" o "bagre anguila" o "resbalosa". Estos organismos son de hábitos gregarios, durante el día se ocultan bajo las piedras y de noche salen a buscar alimentos (Monasterio de Gonzo 2003, Montero \& Autino 2009). Presentan el cuerpo alargado y cilíndrico como una anguila, de allí proviene el nombre popular con el que se lo conoce en Suramérica. La coloración es marrón verdosa en el lomo, aclarándose en la zona ventral. Sujetándose con sus fuertes aletas pectorales, ubicadas cerca de la cabeza, se mantiene cerca de las piedras y troncos, para evitar que lo arrastre la corriente, en las zonas rápidas de los ríos donde le gusta moverse. Alcanzan una longitud de hasta $280 \mathrm{~mm}$. Distribuidos en las cuencas ParanoPlatense, Uruguay y sur de Brasil (Montero \& Autino 2009)

\section{RESULTADOS}

Se capturaron en total 1030 ejemplares de Silurifomes, de los cuales 637 correspondieron a C. paleatus, 262 a T. spegazzinni y 131 a $H$. mustelinus. Las muestras analizadas incluyeron ejemplares capturados en las cuatro estaciones del año.
La cantidad de hembras analizadas, las medidas morfométricas, IGS, fecundidad y el diámetro promedio de los ovocitos se indican en el Cuadro 1.

El análisis del factor de condición $\mathrm{K}$ demostró que existen diferencias significativas $(p=0,0024)$ en los diferentes estadios de madurez de las hembras de T. spegazzinii, estas diferencias se verificaron entre las desovadas en relación a las que están en maduración o maduras, siendo en este último estadio donde se observó el mayor promedio de $\mathrm{K}(0,0017 \pm 0,0002)$. En el caso de $C$. paleatus también se observaron diferencias significativas $(p=0,0312)$ entre las hembras maduras y las desovadas, presentando el mayor valor de $\mathrm{K}$ las desovadas $(0,0049 \pm 0,0007)$. La escasa cantidad de hembras maduras de $H$. mustelinus capturadas no permitió realizar el análisis comparativo. No obstante el K promedio de las maduras fue de 0,0012 ( $\pm 0,0001)$ mientras que las que se encontraban en maduración presentaron un K promedio de 0,0009 ( $\pm 0,0001)$.

\section{Morfología gonadal}

\section{Características macroscópicas}

Las tres especies estudiadas presentan un par de ovarios ubicados en la región dorsal de la cavidad abdominal. Los ovarios inmaduros son de forma aplanada y de coloración blanco-amarillenta, mientras que los ovarios en maduración son más oscuros y piriformes. Ambos se unen por el extremo más angosto, hasta alcanzar el orificio genital. En el período de madurez total los ovocitos

CUADRO 1

Tallas, IGS, fecundidad y diámetro promedio de los ovocitos de las tres especies analizadas.

\begin{tabular}{lccc}
\hline & C. paleatus & T. spegazzinii & H. mustelinus \\
\hline $\begin{array}{l}\text { Sitio de captura de hembras } \\
\text { analizadas }\end{array}$ & $\begin{array}{c}\text { La Ciénega y A } \\
\text { Isasmendi }\end{array}$ & $\begin{array}{c}\text { La Ciénega y } \\
\text { A }^{\circ} \text { Isasmendi }\end{array}$ & $\begin{array}{c}\text { A }^{\circ} \text { Gallinato y } \\
\text { Río Mojotoro }\end{array}$ \\
$\begin{array}{l}\text { Cantidad de hembras } \\
\text { analizadas (N) }\end{array}$ & 51 & 67 & 20 \\
$\begin{array}{l}\text { Talla promedio } \pm \text { desvío } \\
\text { estándar (mm) }\end{array}$ & $31( \pm 5)$ & $42( \pm 13)$ & $60( \pm 35)$ \\
$\begin{array}{l}\text { Talla mínima de madurez } \\
\text { sexual (mm) }\end{array}$ & 27,45 & 30,48 & 60,94 \\
$\begin{array}{l}\text { Cantidad de hembras } \\
\text { maduras analizadas }\end{array}$ & 22 & 21 & 4 \\
$\begin{array}{l}\text { IGS promedio de hembras } \\
\text { maduras } \pm \text { desvío estándar }\end{array}$ & $9,27( \pm 3,09)$ & $9,70( \pm 5,89)$ & $3,87( \pm 2,42)$ \\
$\begin{array}{l}\text { Fecundidad promedio } \\
\begin{array}{l}\text { Diámetro promedio de } \\
\text { ovocitos maduros ( } \mu \text { m) }\end{array}\end{array}$ & 3438 & 772 & 513 \\
& 1250 & 1029 & 1400
\end{tabular}


fueron fácilmente reconocibles. La longitud promedio de los ovarios maduros fue de $9,5 \pm 2,3 \mathrm{~mm}$ en C. paleatus ( $\mathrm{n}=$ $22)$, de $10,8 \pm 5,3 \mathrm{~mm}$ en $T$. spegazzinii $(n=21)$ y de $26,9 \pm$ $9,9 \mathrm{~mm}$ en $H$. mustelinus $(\mathrm{n}=4)$. Es de destacar que en todos los estadíos las gónadas de $H$. mustelinus fueron más grandes que las de las otras especies.

\section{Características microscópicas}

\section{Inmaduras}

Las gónadas inmaduras se caracterizaron por la presencia de numerosos ovocitos previtelogénicos redondeados o poligonales, de núcleos claros con abundantes y numerosos nucléolos periféricos, citoplasma basófilo homogéneo y escaso tejido conectivo dispuesto en bandas. El diámetro mayor promedio de estos ovocitos en C. paleatus fue de 103,58 ( $\pm 28,59) \mu \mathrm{m}$, en $T$. spegazzinii 49,49 $( \pm 8,11) \mu \mathrm{m}$ y en $H$. mustelinus $47,79( \pm 8,97) \mu \mathrm{m}$ (Fig. 2A).

\section{En maduración}

En este estadio, los ovocitos presentaron mayor tamaño que la etapa anterior, siendo evidente la presencia de vesículas o alvéolos en el citoplasma, PAS positivos, considerándose incluso dentro de este estadío aquellos en los que pudo observarse la aparición de esferas o glóbulos con vitelo. Cabe destacar que de las tres especies, T. spegazzinii presentó los alvéolos más claros y grandes. Además, se evidenció en esta etapa el desarrollo de las paredes del folículo, por la diferenciación de la teca externa y la capa de células granulosas. El valor promedio del diámetro mayor de estos ovocitos en C. paleatus fue de 415,77 $( \pm 121,78) \mu \mathrm{m}$, en T. spegazzinii $120,52( \pm 21,94) \mu \mathrm{m}$ y en H. mustelinus 295,99 ( $\pm 86,43) \mu \mathrm{m}$ (Fig. 2B).

\section{Maduras}

En este estadio, es evidente el aumento en el tamaño de los ovocitos. El núcleo, cuando se pudo distinguir, era
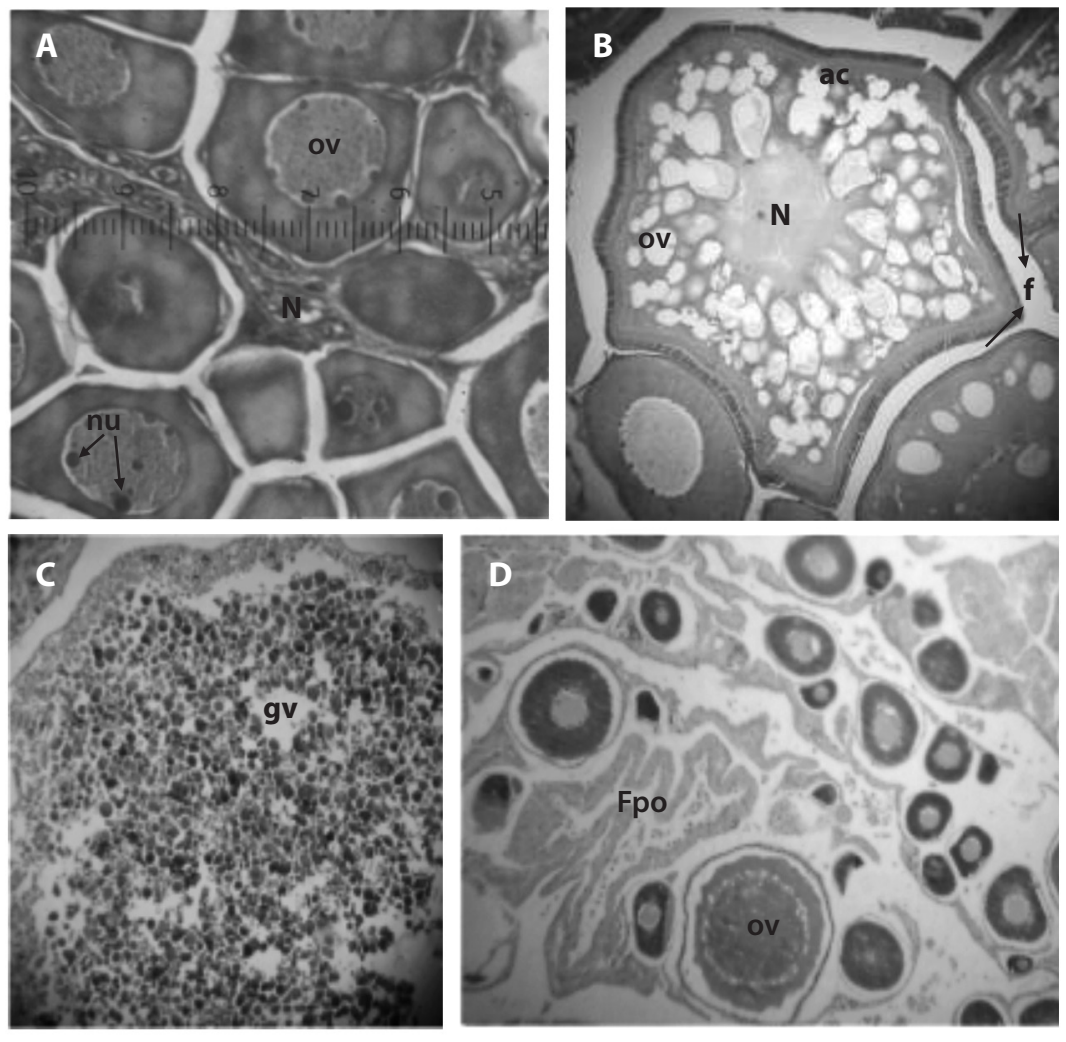

FIG. 2. Fotomicrografías de ovarios en diferentes etapas de maduración. A.Ovario inmaduro de T. spegazzinii, H\&E, 400X. B. Ovario en maduración de T. spegazzinii; PAS, 100X. C. Ovocito maduro de H. mustelinus; H\&E, 50X. D. Ovario desovado de C. paleatus; H\&E, 50X.

ac: alvéolos corticales; f: folículo; fpo: folículo postovulatorio; gv: gránulos de vitelo; N: núcleo; nu: nucléolos; ov: ovocitos. 
excéntrico; se observaron glóbulos de vitelo y vesículas lipídicas, por lo que el citoplasma adquirió un aspecto no homogéneo y granular, dispersándose con facilidad (Fig. 2C). Las membranas que constituyen el folículo se encontraron completamente diferenciadas, por lo que pudieron observarse fácilmente.

Al finalizar esta etapa en los ovocitos se observó una tendencia a deformarse, presentando con frecuencia las membranas foliculares desprendidas, destacándose también la coalescencia de los glóbulos de vitelo y las vesículas lipídicas. En el Cuadro 1 se registran los diámetros promedios en esta etapa.

\section{Desovadas}

El citoplasma de los ovocitos es invadido por las células de la granulosa que digieren el vitelo y finalmente lo degeneran. El aspecto general de la gónada es laxo y se observan numerosas células pequeñas, los espacios vacíos que dejaron los ovocitos maduros y las "marcas de fresa" representadas por los folículos postovulatorios. (Fig. 2d).

\section{Análisis de la etapa de madurez según la estación del año}

El número de hembras capturadas de las tres especies en las diferentes estaciones del año y su correspondiente maduración, está representado en la Fig. 3 ( $A, B$ y C).

Se encontraron hembras de $C$. paleatus en maduración en todas las estaciones del año. En el caso de T. spegazzinii predominaron hembras maduras en las estaciones cálidas (primavera y verano). Respecto a H. mustelinus se observó un claro predominio de hembras en maduración y maduras en primavera.

El análisis de los histogramas de las frecuencias ovocitarias en las hembras analizadas, demuestra una tendencia a considerar a estas especies como desovadoras parciales, ya que los ovocitos maduran sincrónicamente en dos grupos (Fig. 4).

\section{DISCUSIÓN}

De las tres especies de peces Siluriformes analizadas, la mayor cantidad de hembras maduras y desovadas fueron colectadas en primavera y verano, mientras que en las estaciones frías predominaron las inmaduras y en maduración.

En ambos sitios de muestreo (La Ciénaga y arroyo Isasmendi) se capturaron hembras de C. paleatus en maduración durante todas las estaciones y 3 hembras maduras en invierno, por lo que se puede inferir que esta especie
Inmaduro $\square$ En maduración $\square \quad$ Maduro $\square$ Desovado
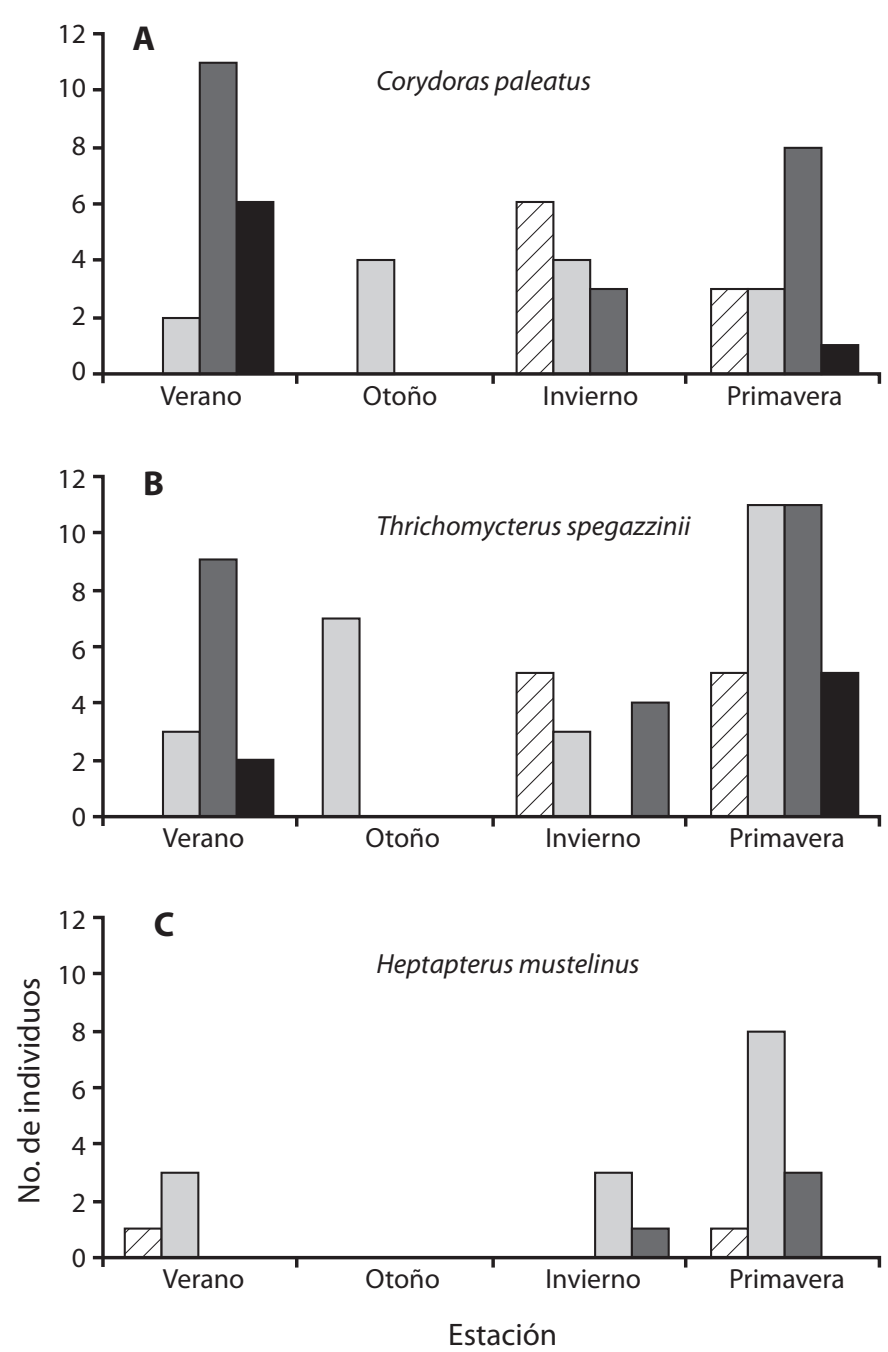

FIG. 3. Estadíos de madurez en cada estación del año, para las tres especies analizadas. A. C. paleatus; B. T. spegazzinii; C. H. mustelinus.

desova en cualquier estación del año, con picos en verano. La talla mínima de madurez sexual de C. paleatus en estos ambientes fue de $27,45 \mathrm{~mm}$. Bervian \& Fontoura (1994) establecieron el tamaño de primera maduración para esta especie en 47,50mm, provenientes de una población lacustre de Brasil en la cual también comprobaron que se reproducen durante todo el año con picos en febrero. Braz de Araujo \& Garutti (2002) informaron para Aspidoras fuscoguttatus, un Siluriforme de bajo porte similar a C. paleatus, una talla media de primera madurez de $37,1 \mathrm{~mm}$. En nuestro estudio esta especie presentó la mayor fecundidad (3438 ovocitos), muy por encima de la reportada por 
Burgess (1989) quien registró de 250 a 400 ovocitos en hembras de C. paleatus criadas en condiciones artificiales.

Respecto a T. spegazzinii, las hembras capturadas en La Ciénaga y arroyo Isasmendi durante la primavera presentaron todos los estadíos de maduración, aunque en otoño e invierno ninguna se encontraba madura, por lo que podemos inferir que el período de desove se encuentra en
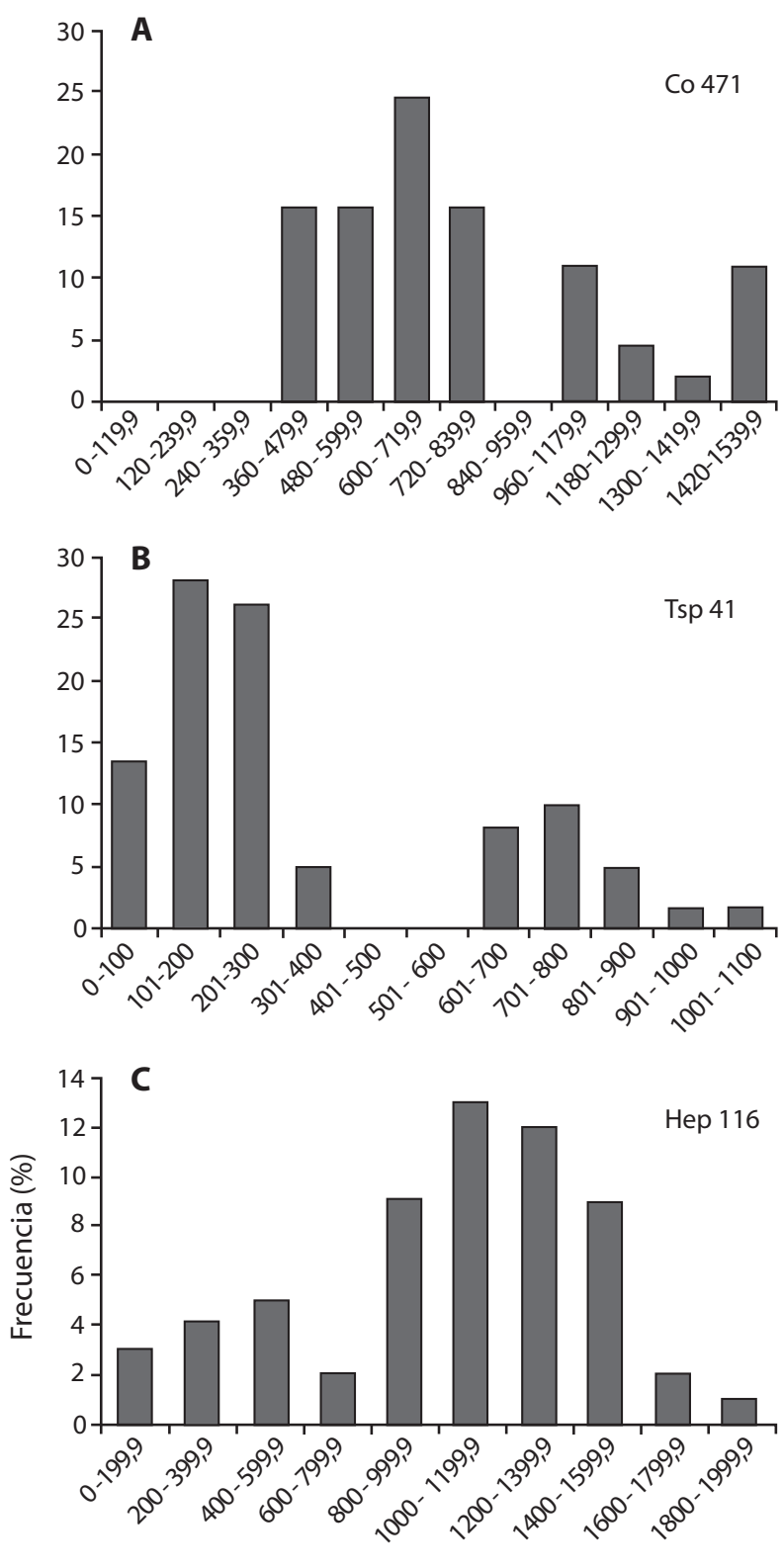

Diámetro ovocitos $(\mu \mathrm{m})$

FIG. 4. Frecuencia ovocitaria correspondiente a tres hembras maduras de las especies analizadas. A. C. paleatus; B. T. spegazzinii; C. H. mustelinus. las estaciones cálidas, coincidiendo con lo observado por Habit et al. (2005) para T. areolatus. Los valores de fecundidad obtenidos (772 ovocitos) fueron muy superiores a los observados por Román-Valencia (2001) para T. caliense, quien reportó valores de 191 ovocitos. El diámetro de los ovocitos fue similar $1029 \mu \mathrm{m}$ para T. spegazzinii y $1150 \mu \mathrm{m}$ para T. caliense. La talla mínima de madurez sexual que obtuvimos fue de 30,48mm, mientras que Román-Valencia (2001) reportó para T. caliense una talla promedio de predesove de $90 \mathrm{~mm}$.

Respecto a $H$. mustelinus, la cantidad de hembras maduras fue escasa. Se capturaron 3 ejemplares maduros en primavera y uno a finales de invierno. Se destaca que éstos ejemplares fueron capturados utilizando caña y anzuelo, en los sitios de mayor profundidad y velocidad de la corriente. La mayoría de los ejemplares capturados correspondieron a individuos juveniles indefinidos $(n=20)$, por lo que podemos inferir que el arroyo El Gallinato sería un sitio de desove y crecimiento de las crías. Destacamos que la fecundidad promedio (513) fue menor que en las otras especies analizadas, sin embargo los ovocitos maduros fueron los de mayor diámetro $(1400 \mu \mathrm{m})$.

Los valores del IGS en las hembras maduras fueron muy similares en C. paleatus y T. spegazzinii, pero en H. mustelinus fue notablemente menor. Esto significaría, por un lado, que esta especie destinaría menos recursos en las gónadas que las anteriores, hecho que también se verifica en la cantidad promedio de ovocitos producidos por la hembra. El factor de condición $\mathrm{K}$ de T. spegazzinii presentó los mayores valores en las hembras maduras. En C. paleatus el valor de $\mathrm{K}$ fue mayor en las inmaduras y luego decreció alcanzando su valor menor en las hembras maduras para volver a aumentar en las desovadas, comportamiento similar se observó en hembras de Imparfinis nemacheir, otro Siluriforme de bajo porte (Román-Valencia \& Hernández 2006), este aumento en las etapas inmaduras sugiere una gran ingesta previa de nutrientes preparándose para el próximo evento reproductivo.

Los histogramas de frecuencia ovocitaria obtenidos en ejemplares correspondientes a las tres especies, indicarían que presentan una tendencia al desarrollo sincrónico de ovocitos por grupo, de acuerdo a lo planteado por Saborido (2004), ya que se observan al menos dos grupos de tamaños de ovocitos al mismo tiempo (bimodal).

Los cambios histológicos en las diferentes etapas de madurez de las gónadas de las tres especies nos permite inferir que se trataría de especies desovadoras parciales. Por otro lado la época de desove de T. spegazzinii y H. mustelinus se extendería desde la primavera temprana hasta mediados de verano, mientras que $C$. paleatus desovaría durante todo el año, con picos en verano. Los ambientes 
muestreados serían adecuados para que se lleve a cabo el ciclo reproductivo de estas especies.

\section{AGRADECIMIENTOS}

Agradecemos a Andrea Guillade por haber revisado y corregido la traducción al inglés del Abstract, al Ing. Miguel Menéndez, de la cátedra Sensores Remotos de la Universidad Nacional de Salta, por haber preparado el mapa y a todos aquellos que nos ayudaron en la recolección de las muestras.

\section{REFERENCIAS}

Agostinho, A., A. Vazzoler \& S. Thomaz. 1995. The high Paraná river basin: limnological and ichtyological aspects, p. 59103, In:Tundisi, J., C.Bicudo, T. Matsumara-Tundisi. Limnology in Brazil. Academy of Sciencies/Brazilian Limnological Society. Brasil.

Bagenal, T. 1978. Aspects of fish fecundity. Ecology of freshwater fish production 4: 75-101.

Bervian, G. \& N. Fontoura. 1994. Dinamica populacional da ictiofauna da lagoa Fortaleza, Cidreira, Rio Grande do Sul. III. Corydoras paleatus (Jenyns, 1842) (Teleostei, Callicthydae). Biociencias 2: 15-23.

Braz de Araujo, R. \& V. Garutti. 2002. Biologia reprodutiva de Aspidoras fuscoguttatus (Siluriformes, Callichthydae) em riacho de cabeceira da bacia do Alto Paraná. Iheringia, Série Zoologia 92: 89-98

Burgess, W.1989. An atlas of freshwater and marine catfishes: a preliminary survey of the Siluriformes. T.F.H. Publications. Neptune City, Nueva Jersey, E.E.U.U.

Calvo, J. \& L.A. Dadone. 1972. Fenómenos reproductivos en el pejerrey (Basilichthys bonariensis) I. Escala y tabla de Madurez. Revista Museo La Plata 11: 153-163.

Habit, E., P. Victoriano \& H. Campos. 2005. Ecología trófica y aspectos reproductivos de Trichomycterus areolatus (Pisces, Trychomycteridae) en ambientes lóticos artificiales. Revista de Biología Tropical 53: 195-210.

Las Heras J. 2010. Corydoras paleatus (http://atlas.drpez.org/ Corydoras-paleatus; consultado 25 de mayo, 2010)

Mc Quinn I.H. \& J. Landy. 1988. Guide de identification microscopique et macroscopique des stades de naturite sexuelle du harong de I'Atlantique (Clupea harengus harengus).
Rapport Technique Canadien des Sciences Halieutiques et Aquatiques: 1655-1670.

Mirande, J.M. \& G. Aguilera. 2009. Los peces de la Selva Pedemontana del noroeste argentino, p. 169-212, In: Brown A.D., P.G. Blendinger, T. Lomáscolo \& P. García Bes (eds) Selva Pedemontana de las Yungas. Ediciones del Subtrópico. Tucumán, Argentina. (http://www.proyungas.org. ar/publicaciones; consultado 25 de mayo, 2010)

Monasterio de Gonzo, G. 2003. Peces de los ríos Bermejo, Juramento y Cuencas Endorreicas de la Provincia de Salta. EUNSA. Salta, Argentina.

Monasterio de Gonzo, G., P. Palavecino \& M.E. Mosqueira. 2008. Vertebrados y Ambientes de la Provincia de Salta. EUNSA. Salta, Argentina.

Montero, R. \& A. Autino. 2009. Sistemática y filogenia de los Vertebrados con énfasis en la fauna argentina. Universidad Nacional de Tucumán. Tucumán, Argentina.

Nikolsky, J.G.V. 1963. The Ecology of Fishes. Academic Journal. Londres, Inglaterra.

Ringuelet, R. 1975. Zoogeografía y ecología de los peces de aguas continentales y consideraciones sobre las áreas ictiológicas de América del Sur. Ecosur 2: 1-122.

Ringuelet, R., R.H. Aramburu \& A.A. Aramburu. 1967. Los peces argentinos de agua dulce. Comisión de investigación científica de la provincia de Buenos Aires. Buenos Aires, Argentina.

Román-Valencia, C. 2001. Ecología trófica y reproductiva de Trichomycterus caliense y Astoblepus cyclopus (Pisces: Siluriformes) en el río Quindio, Alto Cauca, Colombia. Revista de Biología Tropical 49: 657-666.

Román-Valencia, C. \& J. Hernández. 2007. Ecología trófica y reproducción de Imparfinis nemacheir (siluriformes: heptateridae) de la cuenca del río La Vieja, alto río Cauca, Colombia. Dahlia 9: 25-32

Saborido, F. 2004. Ecología de la reproducción y potencial reproductivo en las poblaciones de peces marinos. Curso doutoramento do bienio 2002 - 2004. Instituto de investigaciones Marinas, Universidad de Vigo. Vigo, España.

Vazzoler, A.E. 1996. Biologia da reproduçao de peixes teleósteos: teoria e prática. Maringá, EDUEM. San Pablo, Brasil.

Werner, S.B. 1993. Corydoras. The most armoured catfishes of South America. Dähne Verlag. Ettlingen, Alemania.

Yamamoto, K. 1956. Studies on the formation of fish eggs. Annual cycle in the development of the ovarien eggs in the flounder, Lipsetta oscura. Journal Faculty of Science. Hokkaido University Zoology 12: 362-373. 